\title{
Fundusblutungen bei Malaria tropica
}

\author{
V. Klauß $\beta^{l}$, T. Löscher ${ }^{2}$, M. Kollmann ${ }^{1}$, A. Zarth ${ }^{1}$ \\ 'Universizätsaugenklinik München (Ärztlicher Direktor: Prof. Dr. O.-E. Lund) \\ ${ }^{2}$ Abteilung fur Infektions- und Tropenmedizin der Universität Munchen \\ (Komm. Leiter: Prof. Dr. D. Eichenlaub)
}

Herrn Professor Dr. O.-E. Lund zum 65. Geburtstag gewidmet

\section{Zusammenfassung}

Ein 20jähriger Tourist mit Malaria tropica entwickelte bilaterale prä- und intraretinale Hämorrhagien einschließlich einer Makulablutung mit reversiblem Visusverlust, der während des Rückfluges von Westafrika auftrat. Die Diagnose wurde durch die zunächst aufgesuchte Universitätsaugenklinik eingeleitet. Mögliche Pathomechanismen, okuläre Manifestationen bei Malaria und die zunehmenden Probleme mit importierter Malaria werden zusammenfassend dargestellt und besprochen.

\section{Retinal Hemorrhage in Tropical Malaria}

The case is presented of a 20-year-old tourist with mild falciparum malaria and bilateral preretinal and intraretinal hemorrhage, including a macular hemorrhage with reversible visual loss, which developed during his return flight from West Africa. The diagnosis was initiated by the university eye hospital originally consulted. Possible pathogenic mechanisms, ocular findings in malaria, and the increasing problems of imported malaria are summarized and reviewed.

\section{Kasuistik}

Ein 20jähriger deutscher Tourist war knapp drei Monate durch Westafrika gereist. Während der gesamten Zeit führte er eine regelrechte Malariaprophylaxe mit $2 \times 150 \mathrm{mg}$ Chloroquinbase wöchentlich durch. Unter rezidivierenden, zum Teil auch stärkeren Durchfällen entwickelte er nach ca. 4 Wochen Schüttelfrost, Fieber, Schwächegefühl, Druckgefühl im Epigastrium und Erbrechen. In wechselnder Intensität persistierten diese Beschwerden in den folgenden Wochen bis zur Abreise.

Während des Rückfluges bemerkte der Patient einen plötzlichen Visusabfall mit besonderer Ausprägung links, der hier zu einem verschwommenen Sehen, vor allem zentral, führte.

Kurz nach seiner Ankunft in München wurde er in der Universitätsaugenklinik München vorstellig. Die ophthalmologische Untersuchung erbrachte folgende Befunde:

Der Visus betrug am rechten Auge 0,8 s.c., am linken Auge 0,1 s.c. Die vorderen Augenabschnitte zeigten beidseits anämische Bindehäute und diskret ikterische Skleren. Hornhaut, Vorderkammer, Iris und Pupille waren links und rechts unauffällig. Bei der Fundusskopie zeigten sich am rechten Auge

Klin. Mbl. Augenheilk. 197 (1990) 166-171

(C) 1990 F. Enke Verlag Stuttgart mehrere gefäßnahe intraretinale Blutungen und eine Blutung nahe der regelrechten Papille. Am linken Auge fanden sich eine präretinale Makulablutung sowie ebenfalls periphere intraretinale Blutungen bei regelrechter Papille (Abb. 1a und 2).

Unter dem Verdacht auf eine malariabedingte Fundusblutung wurde der Patient sofort in die Abteilung für Infektions- und Tropenmedizin der Ludwig-Maximilians-Universität München überwiesen. Dort wurden unter anderem folgende Aufnahmebefunde erhoben:

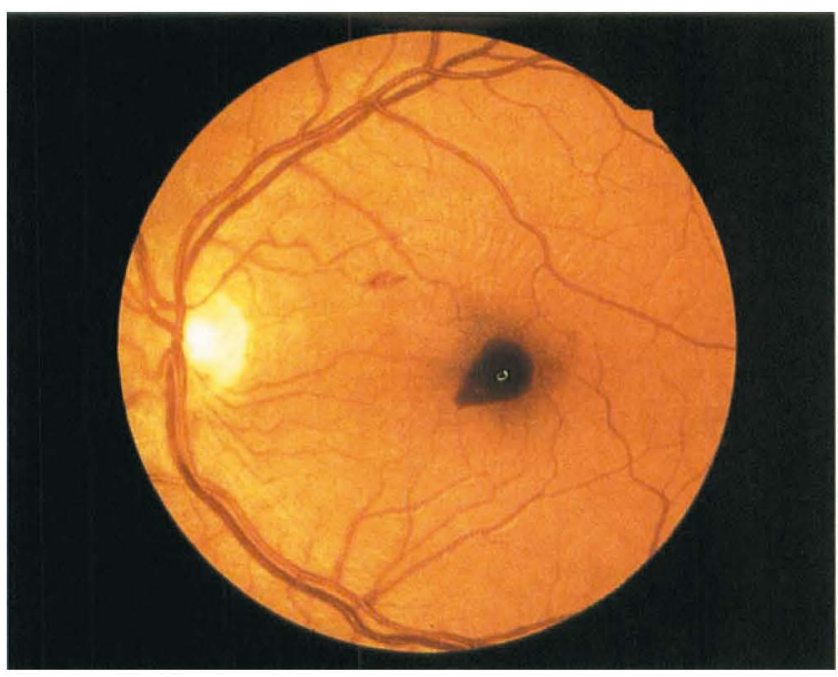

Abb. 1a Präretinale Makulablutung bei Malaria tropica

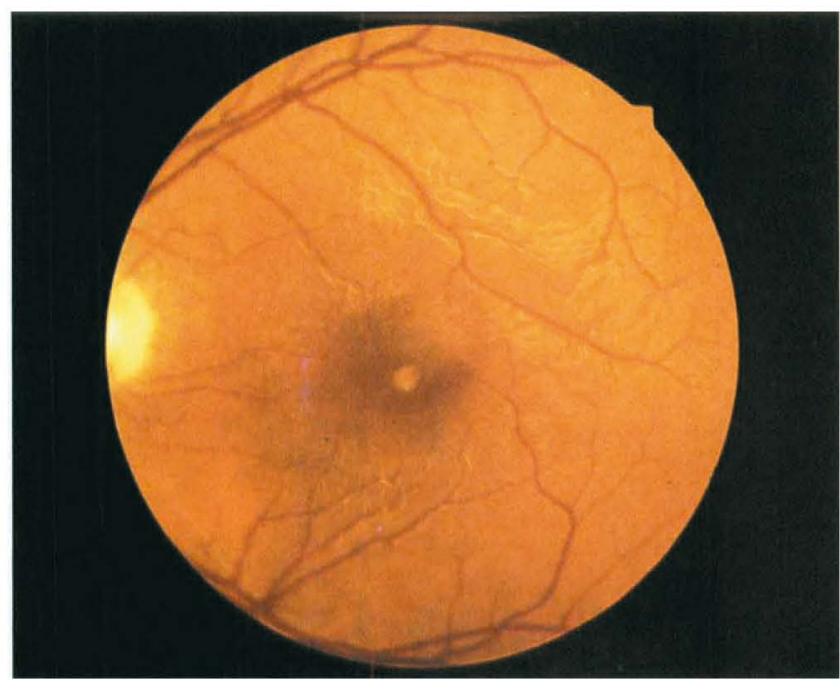

Abb. 1b Zustand nach weitgehender Resorption der präretinalen Makulablutung 4 Wochen nach spontanem Visusabfall (selbes Auge wie in Abb. 1a) 


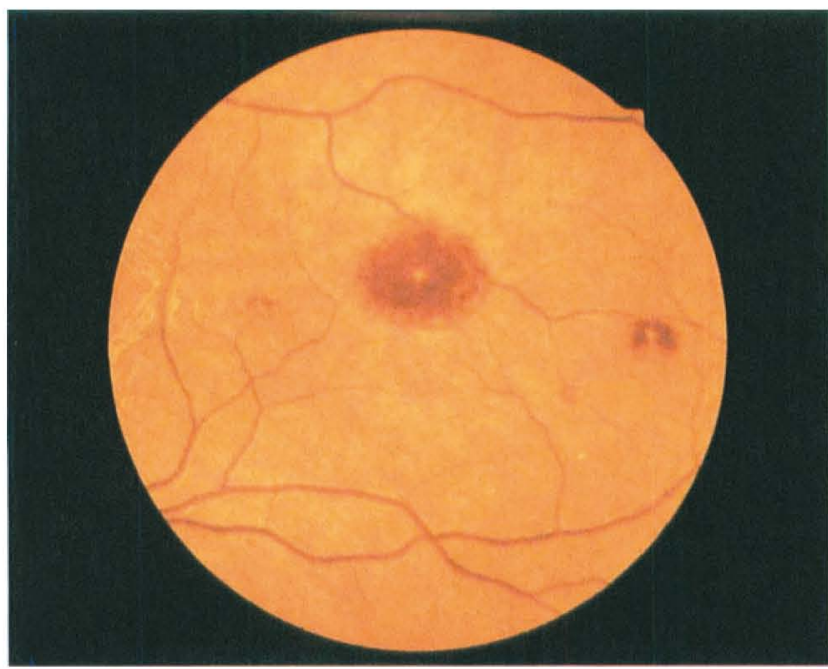

Abb. 2 Periphere intraretinale Blutung bei Malaria tropica

Reduzierter Allgemeinzustand, Größe $190 \mathrm{~cm}$, Gewicht $64 \mathrm{~kg}$, Temperatur $39,2^{\circ} \mathrm{C}$. Der Blutdruck betrug $110 / 60$ $\mathrm{mm} \mathrm{Hg}$, der Puls war $80 / \mathrm{min}$ und unregelmäßig. Die Leberhöhe wurde perkutorisch mit $13 \mathrm{~cm}$ angegeben, die Milz war zwei Querfinger unter dem linken Rippenbogen tastbar und indolent.

Die Laboruntersuchungen ergaben: Erythrozyten 3,9 Millionen/ $\mu \mathrm{l}$, Hämoglobin $11 \mathrm{~g} / \mathrm{dl}$, Hämatokrit 33\%, Thrombozyten $47000 / \mu 1$, Leukozyten $4100 / \mu 1$, Quickwert $78 \%$, PTT 32,7 sec.

Harnstoff, Kreatinin, Leberwerte, Lipase und Urinsediment waren unauffällig, der Hämokkulttest war negativ. Im Blutausstrich fanden sich Trophozoiten von Plasmodium falciparum mit einer Parasitämie von ca. $1 \%$.

Im Oberbauchsonogramm zeigten sich eine leicht vergrößerte Leber und eine deutlich vergrößerte Milz ohne Hinweis auf Milzblutungen. Die Röntgenaufnahme des Thorax erbrachte einen normalen Herz- und Lungenbefund. Das EKG war ebenfalls unauffällig.

Es wurde die Diagnose einer Malaria tropica gestellt. Unter der sofort eingeleiteten stationären Therapie mit Pyrimethamin/Sulfadoxin (Fansidar ${ }^{\circledR}$ ) und einer angesichts der Fundusblutungen durchgeführten parenteralen Chinintherapie trat eine rasche Entfieberung und Besserung des Allgemeinzustandes ein. Die pathologischen Allgemeinbefunde normalisierten sich vollständig. Am zweiten Behandlungstag waren im Dicken Tropfen keine Parasiten mehr nachweisbar.

Bei mehreren augenärztlichen Kontrolluntersuchungen zeigte sich eine allmähliche Resorption der Hämorrhagien. Nach 8 Wochen waren sie nicht mehr nachweisbar, und der Visus lag bei der Abschlußuntersuchung bei 1,0 s.c. beidseits (Abb. 1b).

\section{Malaria}

Mit dem Begriff Malaria wird eine Gruppe von Infektionskrankheiten bezeichnet, die durch Protozoen der Gattung Plasmodium hervorgerufen werden. Es gibt vier humanpathogene Arten, die sich unter anderem morphologisch und in ihrer Klinik unterscheiden:

Plasmodium malariae ist der Erreger der Malaria quartana, Plasmodium vivax und Plasmodium ovale sind die Erreger der Malaria tertiana, und Plasmodium falciparum ist der Erreger der gefährlichsten Form, der Malaria tropica.
Ein Tierreservoir gibt es mit Ausnahme von Plasmodium malariae (Primaten) nicht.

Vektoren sind die Weibchen von über 60 Arten der vor allem dämmerungs- und nachtaktiven Stechmücken der Gattung Anopheles, die in verschiedenen Arten auch in unseren Breiten vorkommen und als Brutplätze stehendes oder sehr langsam fließendes Süß-oder Brackwasser benötigen. Sie sind nicht nur Überträger, sondern die eigentlichen Endwirte, in denen der sexuelle Vermehrungszyklus stattfindet (biologisch-zyklische Ưbertragung). Dafür sind je nach Plasmodienart unterschiedliche Mindesttemperaturen Voraussetzung.

Somit ist die Malariaverbreitung unter anderem vom Vorhandensein bestimmter Biotope mit entsprechenden Temperatur- und Wasserbedingungen abhängig. Zunehmende okologische Bedenken und Insektizidresistenzen erschweren mehr und mehr die chemische Vektorkontrolle $(43,46,54,64,68,76$, 79).

Die Malaria ist eine der häufigsten und bedeutendsten Infektionskrankheiten weltweit., Jährlich erkranken mehrere 100 Millionen Menschen - nach einigen Schätzungen bis zu 400 Millionen (64) - an Malaria, von denen allein in Afrika über 1 Million an Malaria tropica versterben $(10,18,37,69)$.

Gut die Hälfte der Menschheit lebt heute in den vor allem malariabetroffenen Gebieten der Tropen und Subtropen $(75,78)$ (Abb. 3).

Durch den zunehmenden internationalen Reiseverkehr nimmt die Zahl der Erkrankungsfälle auch in Mitteleuropa deutlich zu. So hat sich die Zahl der gemeldeten Erkrankungen in der Bundesrepublik Deutschland in den letzten zwei Jahrzehnten etwa verzwanzigfacht (10). In über $60 \%$ der Einschleppungen aus Afrika handelt es sich um die besonders gefährliche Malaria tropica (Tabelle 1). Alarmierend ist, daß bis zu über $5 \%$ der Erkrankten an ihrer Malaria versterben $(10,56)$.

Das Malariarisiko ist geographisch und jahreszeitlich sehr unterschiedlich. So gibt es z. B. in Äquatornähe über ca. $2500 \mathrm{~m}$ Höhe und in den Subtropen über ca. $1500 \mathrm{~m}$ Höhe in der Regel keine übertragenden Stechmücken und somit keine autochthone Malaria. Im tropischen Afrika ist das Risiko einer Malariaerkrankung besonders hoch. Dabei ist bemerkenswert, daß derzeit fast $90 \%$ der Plasmodium falciparum-Infektionen aus Afrika stammen (19). Als besonders riskante Jahreszeit ist das Ende der Regenzeit anzusehen, da es dann besonders viele Stechmücken gibt $(65,76)$.

Durch die sehr schwierigen sozioökonomischen Bedingungen in einem Großteil der betroffenen Länder, die zunehmenden Insektizidresistenzen der Überträger, die Ausbreitung von Chloroquinresistenzen bei Plasmodium falciparum vor allem in Ost- und Zentralafrika, Asien und zunehmend auch in Westafrika sowie die Zunahme von Resistenzen auch gegen Fansidar ${ }^{(}$ und andere Medikamente werden Malariakontrolle, Prophylaxe und Therapie immer schwieriger $(9,22,24,38,43,52,80)$.

$\mathrm{Zu}$ betonen ist, daß es für die Malaria tropica keine absolut sichere Chemoprophylaxe gibt und somit der Expositionsprophylaxe wesentliche Bedeutung zukommt $(43,65)$. Dazu zählen u.a. geeignete helle, den ganzen Körper bedeckende Kleidung, mückensichere Schlafräume bzw. Moskitonetze sowie die Verwendung geeigneter Insektensprays, mückenvertreibender „mosquito-coils" und Repellents.

Die Inkubationszeiten liegen bei Malaria tropica bei ca. 9-14 Tagen, bei Malaria tertiana bei ca. 12-17 Tagen und bei Malaria quartana bei ca, 3-7 Wochen. Etwas kürzere und deutlich längere Inkubationszeiten sind möglich $(43,45,46,47$, 76). Für die Manifestation der Erkrankung spielen der Immunstatus und der Allgemeinzustand der Infizierten eine wichtige Rolle $(43,76)$. 


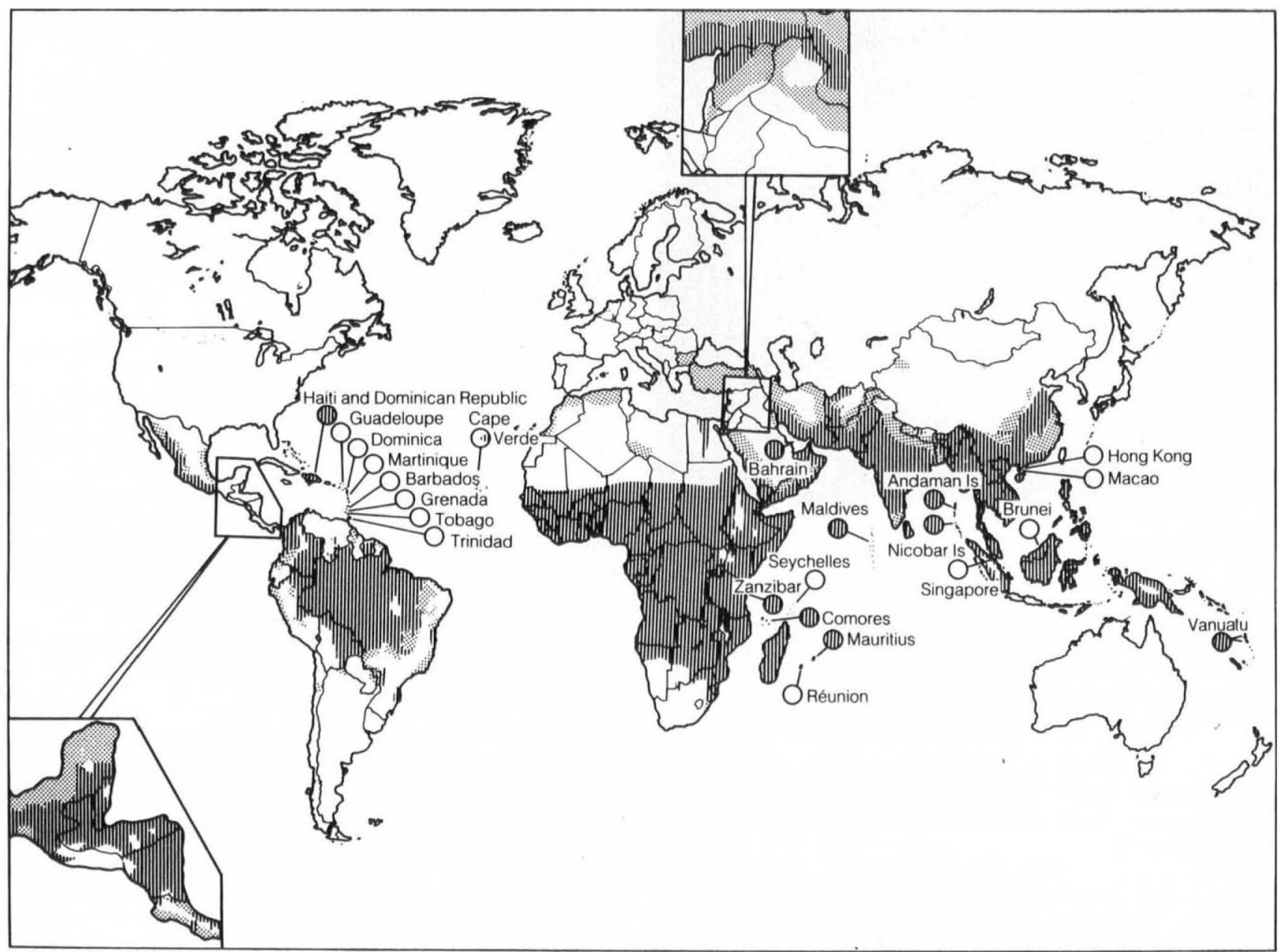

Abb. 3 Geographische Verteilung der Malaria (nach 43)

Malaria - Einschleppungen 1979 - 1988 Bundesrepublik Deutschland und Berlin

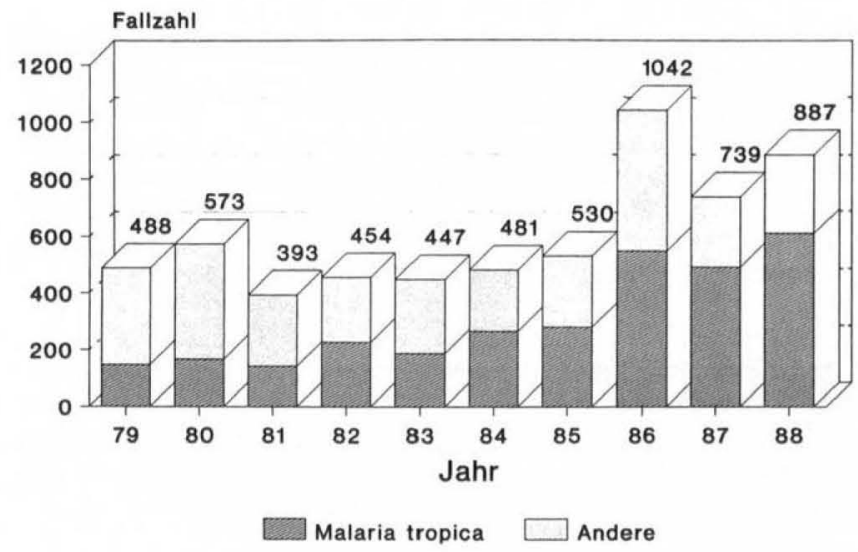

Tab. 1 Eigene Zusammenstellung nach Bundesgesundheitsamt und Pohn $\{55,56\}$
Für die mit importierter Malaria konfrontierten Ärzte, auch Ophthalmologen, wie unser Fall zeigt, ist die Malaria tropica sicher am bedeutsamsten. Gefürchtet ist sie vor allem durch ihren oft foudroyanten Verlauf mit multiplen Organschäden. Die Letalität der unbehandelten Malaria tropica bei Nichtimmunen liegt bei über $20 \%(43,76)$. Dabei stehen klinisch zerebrale, renale und pulmonale Komplikationen im Vordergrund, aber prinzipiell können praktisch alle Organe einschließlich der verschiedenen Augenabschnitte direkt oder indirekt geschädigt werden $(31,43,76)$.

Wesentliche Ursachen der Organschäden sind der Zerfall parasitierter Erythrozyten mit der Freisetzung verschiedener zum Teil ebenfalls schädigender Substanzen und die Veränderung rheologischer Eigenschaften parasitierter Erythrozyten mit sogenanntem „Plugging“ $(16,42,43,76)$. Eine besondere Bedeutung scheint Membranveränderungen parasitierter Erythrozyten zuzukommen, die für das Phänomen der Zytoadhärenz am Kapillarendothel wesentlich sind $(6,35,36,43,47,49,50,58)$. Vor allem die mit einem großen Kapillarsystem versehenen parenchymatösen Organe werden dadurch verstärkt befallen und geschädigt. Bedeutsam ist auch die zum Teil erhebliche Anämie (1, $43,77)$, die wesentlich durch intravasale Hämolyse, vorzeitigen Erythrozytenabbau in der Milz und durch ineffektive Erythropoese bedingt ist. Weiter kommt es unter anderem zu einer Thrombozytopenie $(30,34,43)$, einem prokoagulanten Gerinnungsstatus $(10,15,43)$ [wenn auch extrem selten zu einer klinisch 
manifesten disseminierten intravasalen Koagulation $(19,73)$ ] und einer durch Endothelaktivierung erhöhten Gefäßpermeabilität, durch die es vor allem bei akuter Malaria tropica zu zum Teil erheblichen Flüssigkeitsverschiebungen kommen kann $(19,43,76)$.

Die Parasitämie kann erheblich schwanken und korreliert nicht eng mit der Schwere des klinischen Krankheitsbil-

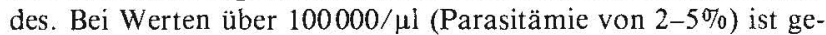
häuft mit Komplikationen zu rechnen $(10,19,43,76)$. Durch die Endothelanheftung parasitierter Eryhtrozyten im Kapillarsystem kann sich dabei eine deutliche Diskrepanz zwischen dem Parasitennachweis im peripheren Blut und dem tatsächlichen Parasitenbefall ergeben $(19,47)$.

\section{Okuläre Manifestationen bei Malaria}

Die Häufigkeit okulärer Beteiligungen bei Malaria wird in der frühen Literatur mit bis über $10 \%$ angegeben $(57,71)$, allerdings zum Teil kontrovers diskutiert (23). Vermutlich sind sie häufiger als bisher angenommen (63).

An den Lidern werden Hyperämie und Ödem beschrieben (31). Die Conjunctiven können hyperämisch sein, petechiale oder flächige Blutungen sowie nach chronisch-rezidivierenden Fieberattacken gelbliche Pigmentierungen (Hämozoin) aufweisen $(31,60)$. Keratitiden, unter anderem als postfebrile Herpes-simplex-Keratitis, sind offenbar häufiger als angenommen, wohingegen Iritiden nur selten gefunden werden $(31,57,60)$.

Als Ausdruck zerebraler Beteiligungen werden Lähmungen der Hirnnerven III, IV und VI, der Akkommodation sowie Nystagmus und eine Argyll-Robertson-Pupille angegeben $(3,7,11,31,60)$.

Beteiligungen des Nervus opticus können sich als Papillitis oder Retrobulbärneuritis manifestieren und offenbar bis zur nachfolgenden Opticusatrophie führen $(11,31,60,70)$. Eine „Malariapapillitis“ mit gräulichen Papillen durch Leukozytenablagerungen wird als pathognomonisch angesehen (60). Eine Amaurosis fugax wird bei komatösen Malaria-Patienten in der Erholungsphase beschrieben (60), weiterhin auch Netzhautödem $(31,41)$ und Papillenödem $(41)$, wie sie vor allem bei Anämie mit erhöhtem intrakraniellen Druck vorkommen (8).

Das Auftreten von Fundusblutungen und Ischämien mit Cotton-wool-Exsudaten in Zusammenhang mit Malaria, vor allem bei Plasmodium falciparum-Infektionen, ist von verschiedenen Autoren dokumentiert worden und findet in letzter Zeit wieder vermehrt Beachtung $(5,14,17,25,28,32,33,39,40,41,51,53,59,60,61,63$, $71,72,74)$.

Die Blutungen sind oft multipel und bilateral $(41,63)$. Vor allem kleinere periphere Hämorrhagien scheinen häufiger als berichtet zu sein und durchaus bei verschiedenen Malariaformen aufzutreten, wohingegen die meist ausgedehnteren Blutungen am hinteren Pol seltener sind, typischerweise bei schweren Plasmodium falciparum-Infektionen vorkommen $(11,14,41,63)$ und zu permanentem Visusverlust führen können (40).
In der Regel werden prä- und intraretinale Blutungen beschrieben, vereinzelt auch subpigmentepithelial-choroidale Blutungen, die aber möglicherweise häufiger als bisher angegeben sind $(32,63)$.

Zumeist wird von malariaassoziierten Fundusblutungen vor allem bei vorbestehender Anämie und zerebraler Beteiligung bei Plasmodium falciparum-Infektionen berichtet. Über die genauen pathogenetischen Mechanismen herrscht noch keine abschließende Klarheit.

Vor allem vaskuläre Schäden und Kapillarverschlüsse u. a. durch Leukozyten oder Emboli parasitierter Erythrozyten $(4,20,51,57,60)$, schwere Anämie (23, $29,31,33)$, Thrombocytopenie $(2,62)$ und weitere Gerinnungsstörungen (41) werden als alleinige Faktoren oder in Kombination diskutiert ebenso wie retinale Angiospasmen (66).

Ergebnisse der Malariaforschung zur Zytoadhärenz parasitierter Erythrozyten bei Malaria tropica wurden ebenfalls in die Diskussion der Pathomechanismen eingeführt (32).

Nach Looareesuwan et al. scheinen Anämie und Thrombozytopenie allgemein eher Indikatoren für eine schwere Malaria tropica zu sein und nicht in alleinigem kausalen Zusammenhang mit malariabedingten Fundusblutungen zu stehen. Nach dieser Studie finden sich Netzhautblutungen vor allem bei Patienten mit schwerer Plasmodium falciparum-Infektion, was sich in Korrelationen zu hoher Parasitämie und Schizontämie sowie erhöhtem Serumkreatinin und erniedrigten AntithrombinIII-Werten ausdrückt (41).

Von mehreren Autoren wird das Auftreten von Netzhautblutungen bei Malaria tropica als vernachlässigtes diagnostisches und prognostisches Kriterium einer eingetretenen oder bevorstehenden zerebralen Malaria bei schwerer Plasmodium falciparum-Infektion angesehen $(41,43,53,60)$.

\section{Diskussion}

Fundusblutungen bei Malaria tropica sind nicht selten und vermutlich von diagnostischer und prognostischer Bedeutung.

In der Regel sind sie assoziiert mit schweren Plasmodium falciparum-Infektionen und zerebralen $\mathrm{Ma}$ nifestationen, denen sie unmittelbar vorausgehen können.

Bei Anämie treten solche Fundusveränderungen gewöhnlich erst bei einer Hämoglobinkonzentration von unter $7 \mathrm{~g} / \mathrm{dl}$ auf - bei Werten von unter $5 \mathrm{~g} / \mathrm{dl}$ sind sie häufig $(13,44,48)$. Typische Befunde sind streifenoder flammenförmige Blutungen, Cotton-wool-Exsudate, geschlängelte Venen und, oft assoziiert mit erhöhtem intrakraniellen Druck, gelegentlich Papillenödeme im Sinne eines Pseudotumor cerebri (8). Thrombocytopenie kann abhängig vom Ausmaß allein oder in Kombination mit Anämie ebenfalls zu Fundusblutungen führen, allerdings erst bei deutlich erniedrigten Werten $(8,21)$. Meist handelt es sich um flächige präretinale Blutungen, die oft im Makula- 
bereich auftreten (13) und damit das zentrale Sehen gefährden können.

Eine klinisch manifeste disseminierte intravasale Koagulation mit Thrombosen vor allem in den submakulären und peripapillären choroidalen Gefäßen und choroidalen Hämorrhagien (8) zeigt sich nur selten. Zumeist findet sich auch bei schwerem Krankheitsbild mit hoher Parasitämie nur ein präkoagulanter Gerinnungsstatus.

Besondere Bedeutung in der Pathogenese der Malaria tropica muß, wie dargestellt, der Interaktion zwischen parasitierten Erythrozyten und Kapillarendothel beigemessen werden. Hierdurch kommt es zu einer relativen Konzentrierung der Parasiten gerade in kapillarreichen Organen. Schädigungen können so z. B. durch rheologische Veränderungen bis zur Stase mit Blutungen und Ischämie und durch Freisetzung toxischer Substanzen eintreten. Malariainduzierte Vaskulopathien durch Entzündungsmediatoren wie $\mathrm{z}$. B. Tumornekrosefaktor scheinen ebenfalls einen wichtigen Mechanismus darzustellen (12, 26, 27).

In dem dargestellten Fall einer Malaria tropica mit multiplen bilateralen intra- und präretinalen Blutungen fand sich bei ausgeprägtem, aber ansonsten ohne Organkomplikationen verlaufendem Krankheitsbild keine wesentliche Anämie, wohingegen die Thrombozytenzahl mit 47000/ $\mu$ l deutlich erniedrigt war. Der Gerinngungsstatus war sonst nicht wesentlich verändert. Die Parasitämie von ca. 1\% ist nicht als ungewöhnlich hoch anzusehen. Eine zerebrale Symptomatik bestand nicht.

Bemerkenswert ist, daß sich die Blutungssymptomatik während des Rückfluges manifestierte.

Auf der Basis der bestehenden Anämie und Thrombozytopenie käme eine Druckänderung mit Verringerung des Sauerstoffpartialdruckes und resultierender relativer Hypoxie als unmittelbar blutungsauslösender Faktor in Betracht.

Inwieweit der Patient möglicherweise in Kürze eine zerebrale Sym ptomatik entwickelt hätte und somit die Fundusblutungen auch im vorliegenden Fall klinisch als Vorläufer einer zerebralen Beteiligung zu bewerten sind, kann aufgrund der sofort eingeleiteten Therapie mit parenteralen Chiningaben nicht beurteilt werden.

Darüber hinaus zeigt dieser Fall, daß auch der Ophthalmologe als zuerst konsultierter Arzt mit einer potentiell lebensbedrohlichen Malariaerkrankung konfrontiert werden kann, bei der einer raschen Diagnose und Therapie wesentliche Bedeutung zukommt. Die Reiseanamnese gab hier den entscheidenden Hinweis zur rechtzeitigen Einleitung weiterer Maßnahmen mit vollständiger Erholung.

Jede fieberhafte Erkrankung während und nach einem Aufenthalt in Risikogebieten kann eine Malaria sein. Auch bei korrekt durchgeführter Prophylaxe muß besonders nach Aufenthalt in endemischen Gebieten unbedingt eine Malaria durch gegebenenfalls auch mehrfach durchzuführende direkte Blutuntersuchungen (Ausstrich und Dicker Tropfen) ausgeschlossen werden $(43,76)$.
Als echtes medizinisches "Chamäleon“ kann sich die Malaria mit den verschiedensten Symptomen manifestieren und somit ohne direkten Erregernachweis erhebliche differentialdiagnostische Schwierigkeiten bereiten. Die Serodiagnostik ist zur Akutdiagnose nicht geeignet $(43,64,67,76)$.

Dem Einbeziehen tropischer Erkrankungen in die differentialdiagnostischen Überlegungen kommt gerade auch angesichts des zunehmenden internationalen Reiseverkehrs wachsende Bedeutung zu.

Die Zahl der.jährlich in der BRD an nicht rechtzeitig erkannter und behandelter Malaria tropica Versterbenden zeigt, daß diesbezüglich noch erheblicher Nachholbedarf besteht.

\section{Danksagung}

Die Autoren danken der Firma Alcan/Thilo, Freiburg, für die Übernahme der Kosten der hier wiedergegebenen Farbabbildungen.

\section{Literatur}

Abdalla, S., D. J. Weatherall, S. N. Wickramasingke et al.: The anaemia of plasmodium falciparum malaria. Brit. J. Haematol. 46 (1980) $171-183$

2 Adelson, E., E. J. Heitzman, J.F. Fennessey: Throbohemolytic thrombocytopenic purpura. Arch. Intern. Med. 94 (1954) 42-60

${ }^{3}$ Agricola, B.: Beobachtungen über Augenerkrankungen bei Malaria. Klin. Mbl. Augenheilk. 58 (1917) 421-430

${ }^{4}$ Anderson, W. A. D.: Pathology. C. V. Mosby Co., St. Louis, 1971

${ }^{5}$ Bell, R. W.: Ophthalmic findings in malaria. Ann. Ophthal. 7 (1975) 1442-1445

${ }^{6}$ Berendt, A. R., D. L. Simmons, J. Tansey, C. J. Newbold, K. Marsh: Intracellular adhesion molecule- 1 is an endothelial cell adhesion receptor for plasmodium falciparum. Nature 341 (1989) 57-59

7 Blatt, N.: Augenveränderungen bei Malaria. Klin. Mbl. Augenheilk. 80 (1928) $468-478$

\$ Bloch, R. S.: Hematologic disorders. In: Duane, T. D., E. A. Jaeger (eds.): Clinical Ophthalmology. Vol. 5, Harper and Row, Philadelphia 1987

${ }^{9}$ Burchard, G. D., R. D. Horstmann, W. H. Wernsdorfer, M. Dietrich: Plasmodium falciparum-malaria: Resistance to Chloroquine, but sensitivity to Mefloquine in the Gabun. A prospective in-vitro study. Tropenmed. Parasitol. 35 (1984) 1-4

${ }^{10}$ Burstedde, V., W. Schneider, F.W. Berges: Malaria-Importe: Ein immer schwierigeres Problem. In: Schneider, W. (Hrsg.): Malaria - Neue Aspekte in Diagnostik, Therapie und Prophylaxe. Editiones (Roche), Basel, 1989

"Bywater, H. H.: Notes on malarial conditions of the eyes. Trans. ophthal. Soc. U. K. 42 (1922) 359-369

12 Clark, $J$. A.: Monokines and lymphokines in malarial pathology. Ann. Trop. Med. Parasitol. 81 (1987) 577-585

${ }^{3}$ Cunningham, R. C.: Retinopathy of blood dyscrasias. In: Duane, T. D., E. A. Jaeger (eds.): Clinical Ophthalmology. Vol. 5, Harper and Row, Philadelphia, 1987

${ }^{14}$ Davis, M. W., A. L. Vaterlaws, J. Simes, P. Torzillo: Retinopathy in malaria. Papua New Guinea med. J. 25 (1982) 19-22

15 Dennis, L. H., J. W. Eichelberger, M. M. Imman, M. E. Conrad: Depletion of coagulation factors in drug-resistant plasmodium falciparum malaria. Blood 29 (1967) 713-721

${ }^{16}$ Desowitz, R. S.: The pathophysiology of malaria after Maegraith. Ann. Trop. Med. Parasitol. 81 (1987) 599-606

${ }^{17}$ Diallo, J. S.: Manifestations ophthalmologiques des parasitoses. Masson, Paris, 1985

18 Dietrich, M.: Malaria. Internist 25 (1984) 203-207

19 Dietrich, M.: persönliche Mitteilung (1989)

${ }^{20}$ Dudgeon, L. S.: A case of malignant malaria. Trans. ophthaimol. Soc. U. K. 41 (1921) 236-238

${ }^{21}$ Duke-Elder, S.: System of Ophthalmology. Mosby, St. Louis, 1967 
22 Eichenlaub, D., H.-G. Hoffmann, G. Rögler, H.-J. Weise: Falciparum-Malaria bei Ostafrika-Touristen trotz Fansidar-Prophylaxe. Dtsch. med. Wschr. 9 (1983) 338-443

${ }^{23}$ Elliot, R. H.: Tropical Ophthalmology. Oxford University Press, London, 1920

${ }^{24}$ Fernex, $M$.: Fortschritte in der Chemoprophylaxe der Malaria. Internist 25 (1984) 208-215

${ }^{25}$ Grant, W. M.: Ocular complications of malaria. Arch. Ophthal. (Chicago) 35 (1946) 48-54

${ }^{26}$ Grau, G. E., P. F. Piquet, P. Vassalli, P.-H. Lambert: Involvement of tumor necrosis factor and other cytokines in immune-mediated vascular pathology. Int. Arch. Allergy. Appl. Immunol. 88 (1989) 34-39

${ }_{27}$ Grau, G. E., T. E. Taylor, M. E. Morlyneux, J. J. Wirima, P. Vassalli, M. Hommel, P.-H. Lambert: Tumor necrosis factor and disease severity in children with falciparum malaria. N. Engl. J. Med. 320 (1989) 1586-1591

${ }^{28}$ Holler, C., R. Solle, F. Pigney et al.: Hémorragies rétiniennes observées au cours de l'évolution d'un paludisme à plasmodium falciparum. Nouv. Press. Méd. 7 (1978) 3554-3556

29 Holt, J. M., E. C. Gordon-Smith: Retinal abnormalities in diseases of the blood. Brit. J. Ophthal. 53 (1969) 145-160

${ }^{30}$ Horstmann, R. D., M. Dietrich, U. Bienzle, H. Rasche: Malaria-induced thrombocytopenia. Blut 42 (1981) 157-164

3 Huismans, H.: Tierische Parasiten des menschlichen Auges. Bücherei des Augenarztes Heft 80, Ferdinand Enke, Stuttgart, 1979

32 Jean, B., J. Seilnacht, H. J. Thiel: Malaria tropica mit Makulablutung. Ophthalmologica (Basel) 195 (1987) 141-144

${ }^{33}$ Kayembe, D., K. Maertens, J. J. De Laey: Complications oculaires de la malaria cérébrale. Bull. Soc. Belge Ophtal. 190 (1980) 53-60

34 Kelton, J. G., J. Kreystone, J. Moore, G. Denomme, E. Tozman, M. Glynn, P. B. Neome, J. Gauldic, J. Jensen: Immune mediated thrombocytopenia of malaria. J. clin. Invest. 71 (1983) 832-836

${ }^{35}$ Kilejian, A., A. Abati, W. Trager: Plasmodium falciparum and plasmodium coatneyi: Immunogenicity of „knob-like protrusions“ in infected erythrocyte membranes. Exp. Parasito. 42 (1977) 157-164

36 Kilejian, A.: Cytoadhaesion. Proc. Natl. Acad. Sci. USA 76 (1979) 4650-4653

${ }^{37}$ Kretschmer, H.: Klinik und Therapie der Malaria. In: Schwick, H. G. (Hrsg.): Tropenkrankheiten. Umwelt und Medizin Verlagsgesellschaft mbH, Frankfurt/Main, 1988

${ }^{38}$ Kroeger, A., H. J. Diesfeld: Medikamentenresistente Malaria in Ostafrika. Dtsch. med. Wschr. 9 (1983) 344-346

39 Lafaix, C., M. Dumas, A. Nouhouayi, M. Rey: Cécité consécutive au paludisme cérebrale (d'après 7 case). Bull. Soc. Méd. Afr. Noire Langue Franc. 15 (1970) 423-428

40 Lagraulet, J. et al.: The ocular complications of malaria. Bull. Soc. Pathol. Exot. 60 (1967) 217-221

${ }^{41}$ Looareesuwan, S., D. A. Warrell, N. J. White et al.: Retinal hemorrhages, a common sign of prognostic significance in cerebral malaria. Amer. J. Trop. Med. Hyg. 32 (1983) 911-915

${ }^{42}$ Maegraith, B.: Other pathological processes in malaria. Bull. Med. Health. Org. 50 (1974) 187-193

${ }^{43}$ Manson-Bahr, P. E. C., D. R. Bell (eds.): Mansons' tropical diseases. Ballière Tindal, London, 1987

${ }^{44}$ Marshall, $R$. A.: A review of lesions in the optic fundus in various diseases of the blood. Blood 14 (1959) 882-891

45 Mehlhorn, H. (Hrsg.): Parasitology in focus. Springer, Heidelberg, 1988

${ }^{46}$ Mehlhorn, H., G. Piekarski: Grundriß der Parasitenkunde. G. Fischer, Stuttgart, 1989

${ }^{47}$ Mehlhorn, H.: Der Entwicklungszyklus der Malaria-Erreger des Menschen. In: Schneider, W. (Hrsg.): Malaria - Neue Aspekte in Diagnostik, Therapie und Prophylaxe. Editiones $\langle$ Roche〉, Basel, 1989

${ }^{48}$ Merin, S., M. Freund: Retinopathy in severe anaemia. Amer. J. Ophthal. 66 (1968) 1102-1106

${ }^{49}$ Ockenhouse, C. F., N. N. Tandon, C. Magowan et al.: Identification of a platelet membrane glycoprotein as a falciparum malaria sequestration receptor. Science 243 (1989) 1469-1471

so Oquendo, P., E. Hundt, J. Lawler, B. Seed: CD 36 directly mediates cytoadherence of plasmodium falciparum parasitized erythrocytes. Cell 58 (1989) 95-101

51 Pereyra, G.: Emorragie retiniche de malaria. Arch. Ottal. (Napoli) 29 (1922) 49-79

52 Peters, $W$.: Chemotherapy and drug resistance in malaria. Academic Press, London, 1987
${ }^{53}$ Peters, W., H. M. Gilles: A colour atlas of tropical medicine and parasitology. Wolf Medical Publications Ltd., London, 1989

${ }^{54}$ Piekarski, G.: Medizinische Parasitologie. Springer, Heidelberg, 1988

${ }^{55}$ Pöhn, H.-P.: persönliche Mitteilung (1989)

56 Pöhn, H.-P., R. Großmann: Malariaeinschleppungen 1980-1986 in die Bundesrepublik Deutschland einschl. Berlin (West). Bundesgesundhbl. Nr. 31 (1988) 2-10

57 Poncet, F.: De la retino-choroidite palustre. Ann. oculist (Paris) 79 (1879) 201-218

${ }^{58}$ Roberts, D. D., J. A. Sherwood, S. L. Spitalnik et al.: Thrombospondin binds falciparum malaria parasitized erythrocytes and may mediate cytoadherence. Nature 318 (1985) 64-66

59 Robertson, J. N.: Ophthalmologic lesions encountered in the tropics with special reference to the ocular manifestations of malaria. North Carolina Med. J. 5 (1944) 483-489

${ }^{60}$ Rodger, F. C.: Eye diseases. In: Manson-Bahr, P. E. C., D. R. Bell (eds.): Mansons' tropical diseases. Ballière Tindal, London, 1987

${ }^{61}$ Ross, J. V. M.: Ocular complications associated with malaria. Eye Ear Nose Throat Mon. 32 (1953) 707-710

${ }^{62}$ Rubenstein, R. A., M. Yanoff, D. M. Albert: Thrombocytopenia, anaemia, and retinal hemorrhage. Amer. J. Ophthal. 65 (1968) 435-439

63 Runyan, T. E., R. C. Ostberg: An unusual macular lesion associated with malaria. Ann. Ophthal. 9 (1977) 1521-1525

${ }^{64}$ Schneider, $\boldsymbol{W}$. (Hrsg.): Malaria - Neue Aspekte in Diagnostik, Therapie und Prophylaxe. Editiones 〈Roche〉, Basel, 1989

${ }^{65}$ Schneider, W.: Mensch, Mücke, Malaria: Wenig Wissen über die wichtigste Seuche der Welt. In: Schneider, W. (Hrsg.): Malaria - Neue Aspekte in Diagnostik, Therapie und Prophylaxe. Editiones (Roche), Basel, 1989

66 Sedan, J.: Temporary blindness caused by retinal angiospasm with malarial origin. Ann. Ocul. 166 (1929) 705-709

${ }^{67}$ Seitz, H. M.: Serologie und künftige Entwicklungen in der Malaria Diagnostik. In: Schneider, W. (Hrsg.): Malaria - Neue Aspekte in Diagnostik, Therapie und Prophylaxe. Editiones (Roche), Basel, 1989

68 Service, M. W.: Blood-sucking insects: Vectors of disease. Edward Arnold, London, 1986

${ }^{69}$ Spencer, H. C., G. T. Strickland: Malaria. In: Strickland, G. T. (ed.): Tropical medicine. Saunders, Philadelphia, 1984

70 Stachan, H.: On a form of multiple neuritis prevalent in the West Indies. Practioner 59 (1897) 477-484

7 Sulzer, D. E.: Troubles de la vision dans la paludisme. Arch. Ophtal. (Paris) 10 (1890) 193-208

72 Villard, H.: Les complications oculaires du paludisme. Arch. Ophtal. (Paris) 47 (1930) 200-208

73 Vreeken, J., T. M. Cremer-Goote: Haemostatic defect in non-immune patients with falciparum malaria: no evidence of diffuse intravascular coagulation. Brit. med. J. 2 (1978) 533-535

${ }^{74}$ Werner, H.: Über Netzhautblutungen bei Malaria. Arch. Schiffs- und Tropen. Hyg. 15 (1911) 431-435

75 Wernsdorfer, W. H.: Malaria: Epidemiologie, Resistenzprobleme, Prophylaxe. In: Schick, H. G. (Hrsg.): Tropenkrankheiten. Umwelt und Medizin Verlagsgesellschaft mbH, Frankfurt/Main, 1988

76 Wernsdorfer, W. H., J. McGregor (eds.): Malaria - principles and practice of malariology. Churchill Livingston, London, 1988

77 Woodruff, A. W., V.E. Ansdell, L. E. Pettitt: Cause of anaemia in malaria. Lancet 1 (1979) 1055-1057

${ }_{78}$ World Health Organisation: World malaria situation 1985. Malaria action programm. Wid. hlth. statist. quart. 40 (1987)

79 World Health Organisation: Resistance of vectors of disease to pesiticides. Fifth report of the WHO Expert Committee on vector biology and control. WHO Technical Report Series 655 (1980)

${ }^{80}$ World Health Organisation: Advances in malaria chemotherapy: report of WHO Scientific Group. WHO Technical Series 711 (1984)

Manuskript erstmals eingereicht 27. 12. 1989, zur Publikation in der vorliegenden Form angenommen 17. 1. 1990

\section{Dr. M. Kollmann}

Universitätsaugenklinik München

Mathildenstr. 8

8000 München 2, BRD 